\title{
Bacterial community dynamics during embryonic development of the little skate (Leucoraja erinacea)
}

\author{
Katelyn Mika ${ }^{1,2^{*}+}$, Alexander S. Okamoto ${ }^{3 \dagger}$, Neil H. Shubin ${ }^{1}$ and David B. Mark Welch ${ }^{4}$
}

\begin{abstract}
Background: Microbial transmission from parent to offspring is hypothesized to be widespread in vertebrates. However, evidence for this is limited as many evolutionarily important clades remain unexamined. There is currently no data on the microbiota associated with any Chondrichthyan species during embryonic development, despite the global distribution, ecological importance, and phylogenetic position of this clade. In this study, we take the first steps towards filling this gap by investigating the microbiota associated with embryonic development in the little skate, Leucoraja erinacea, a common North Atlantic species and popular system for chondrichthyan biology.

Methods: To assess the potential for bacterial transmission in an oviparous chondrichthyan, we used 16S rRNA amplicon sequencing to characterize the microbial communities associated with the skin, gill, and egg capsule of the little skate, at six points during ontogeny. Community composition was analyzed using the QIIME2 pipeline and microbial continuity between stages was tracked using FEAST.
\end{abstract}

Results: We identify site-specific and stage-specific microbiota dominated by the bacterial phyla Proteobacteria and Bacteroidetes. This composition is similar to, but distinct from, that of previously published data on the adult microbiota of other chondrichthyan species. Our data reveal that the skate egg capsule harbors a highly diverse bacterial community-particularly on the internal surface of the capsule-and facilitates intergenerational microbial transfer to the offspring. Embryonic skin and external gill tissues host similar bacterial communities; the skin and gill communities later diverge as the internal gills and skin denticles develop.

Conclusions: Our study is the first exploration of the chondrichthyan microbiota throughout ontogeny and provides the first evidence of vertical transmission in this group.

Keywords: Microbial transmission, Egg capsule, Chondrichthyes, Rajidae, Oviparity

\section{Introduction}

Host-associated microbial communities are often species and tissue-specific due to complex local interactions between hosts and microbes [1,2]. Species can acquire

\footnotetext{
*Correspondence: kmmika@uchicago.edu

†Katelyn Mika and Alexander S. Okamoto have contributed equally to this work

${ }^{1}$ Organismal Biology and Anatomy, University of Chicago, 900 E 57th St, Culver Hall 108 OBA, Chicago, IL 60637-1428, USA

Full list of author information is available at the end of the article
}

their microbiota through three possible processes: horizontal microbial transmission between conspecifics, vertical microbial transmission from parents to offspring, or similar environmental sourcing across the host species [3]. In the first case, microbes can be horizontally transferred between conspecifics during social interactions and sexual behaviors, potentially homogenizing the bacterial communities across the host population. Alternatively, vertical transmission allows parents to directly provide their progeny with symbiotic microbial taxa [4]. Lastly, microbes can be recruited from the surrounding

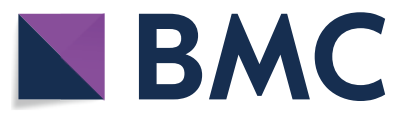

(c) The Author(s) 2021. Open Access This article is licensed under a Creative Commons Attribution 4.0 International License, which permits use, sharing, adaptation, distribution and reproduction in any medium or format, as long as you give appropriate credit to the original author(s) and the source, provide a link to the Creative Commons licence, and indicate if changes were made. The images or other third party material in this article are included in the article's Creative Commons licence, unless indicated otherwise in a credit line to the material. If material is not included in the article's Creative Commons licence and your intended use is not permitted by statutory regulation or exceeds the permitted use, you will need to obtain permission directly from the copyright holder. To view a copy of this licence, visit http://creativecommons.org/licenses/by/4.0/. 
environment through the host's contact with fluids, substrates, or diet, allowing for rapid changes in community composition in response to external conditions over the lifespan of an individual. The relative contributions of these transmission modes likely covary with life history, balancing the need for intergenerational continuity of genomic information with the capacity to respond to environmental cues $[3,5]$.

Next-generation sequencing has facilitated the characterization of a broad range of microbiomes across an increasing diversity of host species; nonetheless, many important marine clades remain understudied. Chondrichthyans-the earliest branching of the extant, jawed-vertebrate lineages -constitute one of the major divisions of vertebrates [6]. To date, only a limited number of culture-independent studies of chondrichthyan microbiota have been conducted [7-13]. Existing studies of chondrichthyans all focus on species belonging to subclass Elasmobranchii, which includes sharks, skates, rays, and guitarfish. This literature is skewed towards the skin or gut microbiota of pelagic sharks $[7-11,13]$ or the skin microbiota of select ray species [11, 12]. These datasets show that elasmobranch skin microbiota differ from that of the surrounding environment and are primarily dominated by the phyla Proteobacteria and Bacteroidetes, similar to the skin microbiota of other marine species [14-16]. However, this work is limited to adult elasmobranchs, providing no direct information on juvenile microbiota or intergenerational transmission in chondrichthyans.

In some clades, microbiome composition closely tracts host phylogeny over evolutionary time, resulting in longterm eco-evolutionary relationships known as phylosymbiosis [17]. Previous research has identified signatures of phylosymbiosis in elasmobranchs by showing a correlation between host phylogenetic distance and the taxonomic composition of the microbiota [11]. Of the three processes of transmission described, horizontal transmission has the most limited explanatory potential for this finding as chondrichthyan species are largely asocial with aggregations driven primarily by environmental factors or reproduction $[18,19]$. Vertical transmission or environmental sourcing are more promising potential mechanisms to explain the signature of phylosymbiosis. Data on environmental sourcing in chondrichthyans are limited $[9,12]$ and are difficult to acquire, while vertical transmission has been unexplored. Given the lack of data and the hypothesis that vertical transmission is widespread in vertebrates [4], we investigated the potential for vertical transmission in the model chondrichthyan, the little skate (Leucoraja erinacea).

Oviparity is present in almost half of chondrichthyans and may be the plesiomorphic reproductive mode for this clade [6, 20]. Like other skates (family: Rajidae), little skates are egg-laying elasmobranchs that protect their embryos inside egg capsules, colloquially known as a mermaid's purses [21]. Development of the egg capsule starts in the nidamental organ where the posterior half is formed before the fertilized egg is deposited into the capsule, at which point the capsule is rapidly sealed shut [22]. These capsules are then laid on the seafloor and the embryos develop inside for months to years depending upon the temperature $[23,24]$ and species [25]. While egg capsules can osmoregulate at all stages, they are initially sealed to anything larger than small molecules, e.g. glucose and urea can pass through but insulin cannot [26, 27]. Slits at the anterior and posterior ends of egg capsule open up late in development allowing seawater to flow through [28]. The potential effects of this environmental shift on the microbiota and host development are unknown. Upon hatching, juvenile skates are self-sufficient, with no known parental care [6]. These life history traits-long embryonic development and lack of parental care after oviposition-pose potential obstacles to vertical microbial transmission in members of this clade. Thus, skates are a useful system for testing vertical transmission because confounding parental contact is minimized and any transmitted microbial community is likely stable over a substantial period of time.

The little skate is a model system for research in chondrichthyan embryology and development [24, 29-32] with a sequenced genome [33]. This species is common in the North Atlantic [34] and easy to obtain through sampling and breeding methods implemented in that region. Little skate embryos have gestational periods inside the egg capsule of 22-54 weeks depending on the season [23]. Embryogenesis is divided into thirty-three stages, based on morphological features [35, 36]. In this study, our goal was to characterize the microbiota associated with embryonic development of the little skate and to assess the potential for vertical transmission in this species. To accomplish this, we used 16S rRNA amplicon sequencing to describe and track changes in bacterial diversity throughout little skate ontogeny by sampling the microbiota of the skin and gills, as well as the internal liquid and internal surface of the egg capsule at six developmental stages. These stages are (i) stage 0 , when capsules are freshly laid and fertilization cannot be visually confirmed; (ii) stage 16, an early stage when the embryo can be visually identified; (iii) stage 26 , by which external gill filaments have formed and the egg capsule is still sealed; (iv) stage 30, when the egg capsule starts to open and the gills remain external; (v) stage 33 , by which time the egg capsule is open and the embryo is fully formed with internal gills; and (vi) adult. These stages span the duration of embryonic development from shortly after 
oviposition until just before hatching and capture distinct periods related to organogenesis and environmental exposure.

\section{Materials and methods Sample collection}

Adult skates used in this study were wild caught in the Northern Atlantic and housed in $15^{\circ} \mathrm{C}$ filtered seawater (400-micron mesh followed by sand filtration) at the Marine Resources Center (MRC) of the Marine Biological Laboratory, Woods Hole, MA. All embryos were laid within the facility tanks by this adult population. Samples were collected from adult females $(n=4)$ by separately swabbing the gills and the skin around the cloaca. Adult females were not directly associated with any particular egg capsule used in this study but were all sexually mature, housed in the MRC breeding tanks, and thus serve as representative, potential mothers. Egg capsules were sampled at five timepoints: stages $0,16,26,30$, and $33(n=4$ each, $n=20$ total $)$ as per refs. [35, 36]. Capsules were windowed with a razor blade and the embryos euthanized by cervical transection. At each stage, samples of the internal liquid $(n=20)$ were collected using a $1000 \mathrm{~mL}$ pipette and the inside of the egg capsule was swabbed $(n=20)$, as shown in Additional file 4: Fig. 1 . All samples at stage 33 were open, as were two samples (A \& D) at stage 30 . All other samples were closed. Samples where the egg capsule slits were already open to the environment were drained into a collection tube before the egg capsule was windowed. At stages 26, 30 and 33, gill filament samples and tail clippings $(\sim 2 \mathrm{~cm}$ long $)$ were collected ( $n=4$ each, $n=24$ total). Control samples included hand swabs of A.S.O and K.M. and bench swabs before sample processing both on the day of sample collection and again on the day of DNA extraction $(n=6$ total). A sample of the bench after sterilization $(n=1)$ was taken on the day of sample collection as well. Sterile water was collected as a negative control. To broadly sample the marine bacteria likely to be encountered by skates in the MRC, $1 \mathrm{~mL}$ of water was collected in $1.5 \mathrm{~mL}$

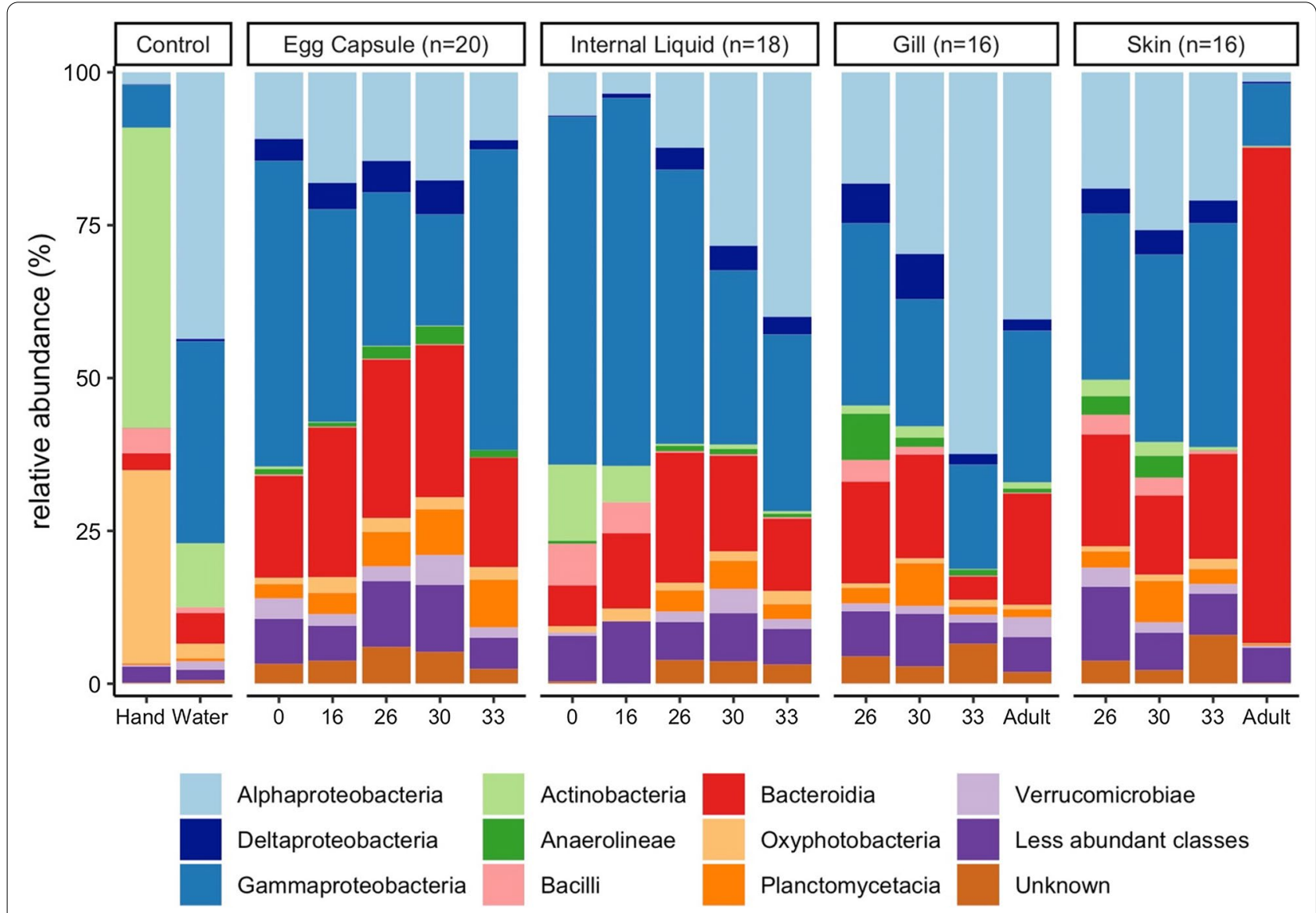

Fig. 1 Taxonomic composition of embryonic and adult skate bacterial communities. Relative abundance of the top ten bacterial classes in the dataset are shown for each site and timepoint as well as for water and hand controls. First, classes of phylum Proteobacteria are shown in shades of blue, followed by other classes ordered alphabetically. For the controls, $n=4$ for hand and $n=8$ for water samples 
tubes from (i) ambient-temperature water tanks $(n=2)$; (ii) $15^{\circ} \mathrm{C}$ tanks housing the skates $(n=2)$; (iii) ocean water from the dock neighboring the pump into the MRC $(n=2)$; and (iv) the bucket used to transfer the skate embryos from the MRC to the dissection station was collected before $(n=1)$ and after sampling $(n=1)$. Prior to sample collection, the bench and all dissection tools were sterilized using Clorox bleach, followed by $70 \%$ ethanol. These surfaces were re-sterilized with $70 \%$ ethanol between each egg capsule and with bleach and ethanol between every four egg capsules. All skate samples were collected on the same day and egg capsules were opened in a randomly selected order.

The DNeasy PowerSoil Kit (Qiagen, Hilden Germany) was used for isolation of the microbial DNA from each sample. FLOQSwabs (COPAN, Murrieta CA) were used to collect all swab samples. Swabs trimmed to fit or tissue samples from the gills and tail were placed directly in the PowerSoil Kit PowerBead tubes after collection. For all liquid samples, $200 \mu \mathrm{L}$ of the sample was added to the corresponding tube. After collection, samples were left at $-20^{\circ} \mathrm{C}$ overnight prior to completion of the extraction protocol. Extraction continued according to the manufacturer's instructions. The negative control and postcleaning bench swab failed to amplify, suggesting our sterilization technique was effective (Additional file 1: Table S1). Pre-cleaning bench samples were thus unlikely to have contaminated other samples and were excluded from further analysis.

\section{Sequencing and library preparation}

To identify the bacterial community within each sample, the V4-V5 region of the $16 \mathrm{~S}$ gene was amplified and sequenced at the Keck Environmental Genomics Facility at the Marine Biological Laboratory as described [37]. Amplification was done using forward primer (518F) CCAGCAGCYGCGGTAAN and reverse primers (926R) CCGTCAATTCNTTTRAGT, CCGTCAATTTCTTTG AGT, and CCGTCTATTCCTTTGANT. Three reverse primers were used to capture known $16 \mathrm{~S}$ sequence diversity more effectively than a single highly degenerate primer. PCR cycle structure was $94^{\circ} \mathrm{C}$ for $2 \mathrm{~min}, 30$ cycles of repeating $94^{\circ} \mathrm{C}$ for $30 \mathrm{~s}, 57^{\circ} \mathrm{C}$ for $45 \mathrm{~s}$, and $72^{\circ} \mathrm{C}$ for $1 \mathrm{~min}$, followed by $72^{\circ} \mathrm{C}$ for $2 \mathrm{~min}$ then a hold at $4^{\circ} \mathrm{C}$. Sequencing was done on an Illumina MiSeq platform. Results were then uploaded to the Visualization and Analysis of Microbial Population Structures (VAMPS) website (https://vamps2.mbl.edu/) [38]. Raw data was obtained and deposited at the NCBI Short Read Archive (PRJNA688288).

Denoising to address amplicon errors, classifying to identify the taxonomic affiliation of each sequence, and alpha and beta diversity methods to assess community composition were all implemented using QIIME2 version 2019.10 [39]. Demultiplexed paired-end sequencing data was denoised without any trimming and chimeras removed using the DADA2 QIIME2 plugin [40]. The resulting amplicon sequence variants (ASVs) were classified by training a Naive Bayes classifier using the SILVA (132 release) $16 \mathrm{~S}$ only, 99\% identity clustered sequences [41-43]. ASVs were collapsed to a maximum specificity of seven taxonomic levels, which corresponds to the species level. Data were normalized using transform_sample_counts in Phyloseq $[44,45]$ by dividing the count per ASV within a sample by the total count for that sample, followed by multiplying this ratio by 10,000 .

\section{Microbial community analysis}

QIIME2 was used to calculate Pielou's evenness, Shannon and Chao1 alpha diversity indices [46], and the Bray-Curtis dissimilarity index for beta diversity [47]. Beta diversity differences between sample types were visualized using principal coordinate analysis. Betweengroup significance levels for alpha and beta diversity were assessed using Kruskal-Wallis [48] and PERMANOVA [49] tests, respectively, with a Benjamini-Hochberg false discovery rate (FDR) correction. The significance threshold for these tests was set at $\mathrm{q}<0.05$. Taxa comprising the common core microbiota [50] of the (i) egg capsule, (ii) combined external gill and embryonic skin, (iii) internal gill, and (iv) adult skin were identified using the featuretable core-features function in QIIME2 at a threshold of $75 \%$ presence. This threshold was chosen to prioritize taxa with high levels of occupancy in tissues of interest. Given our small sample size for each tissue and stage, thresholds higher than $75 \%$ result in very few core taxa, while lowering this threshold results in rapid increases in core taxa numbers.

LEfSe (Linear Discriminant Analysis (LDA) Effect Size) was used to identify significantly enriched taxa and implemented in the Galaxy web application (http://hutte nhower.org/galaxy/) [51] using a $P$-value cut-off of 0.05 , an LDA score cut-off of 2, and a one-against-all strategy. FEAST [52] in R v3.6.2 [53] was used to track bacterial community continuity between stages, with samples from the preceding stage (unless otherwise noted), water, and the investigators' hands used as potential sources. $\mathrm{R}$ Studio (Version 1.2.5033) with the ggplot2 package [54] was used to produce all figures.

\section{Results}

Eighty-four out of eighty-eight samples successfully amplified and were sequenced to produce a total of 3,516,842 reads and 41,486 ASVs (Additional file 1: Table S1 and Additional file 2: Table S2). These ASVs were classified into 2,255 unique taxonomic identities 
using the SILVA database. Each sample contained between 170 and 147,651 reads, with a median value of 36,480. ASV assignments ranged from 1 to 33,211 reads, with a median value of 29. Rarefaction curves of ASVs recovered versus sequencing depth showed that sequencing depth was sufficient to discover the majority of ASVs in a sample (Additional file 4: Fig. 2).

\section{Taxonomic characterization of $L$. erinacea microbiota}

Skate and egg capsule samples are dominated by ASVs of the phyla Proteobacteria (58\% of ASVs), Bacteroidetes (21\%), and Planctomycetes (5\%). The dominant classes identified are Gammaproteobacteria (33\%), Alphaproteobacteria (21\%), Bacteroidia (21\%), and Planctomycetacea (4\%) (Fig. 1). Adult skin samples are uniquely enriched for Bacteroidetes, which accounts for $81 \%$ of the bacterial

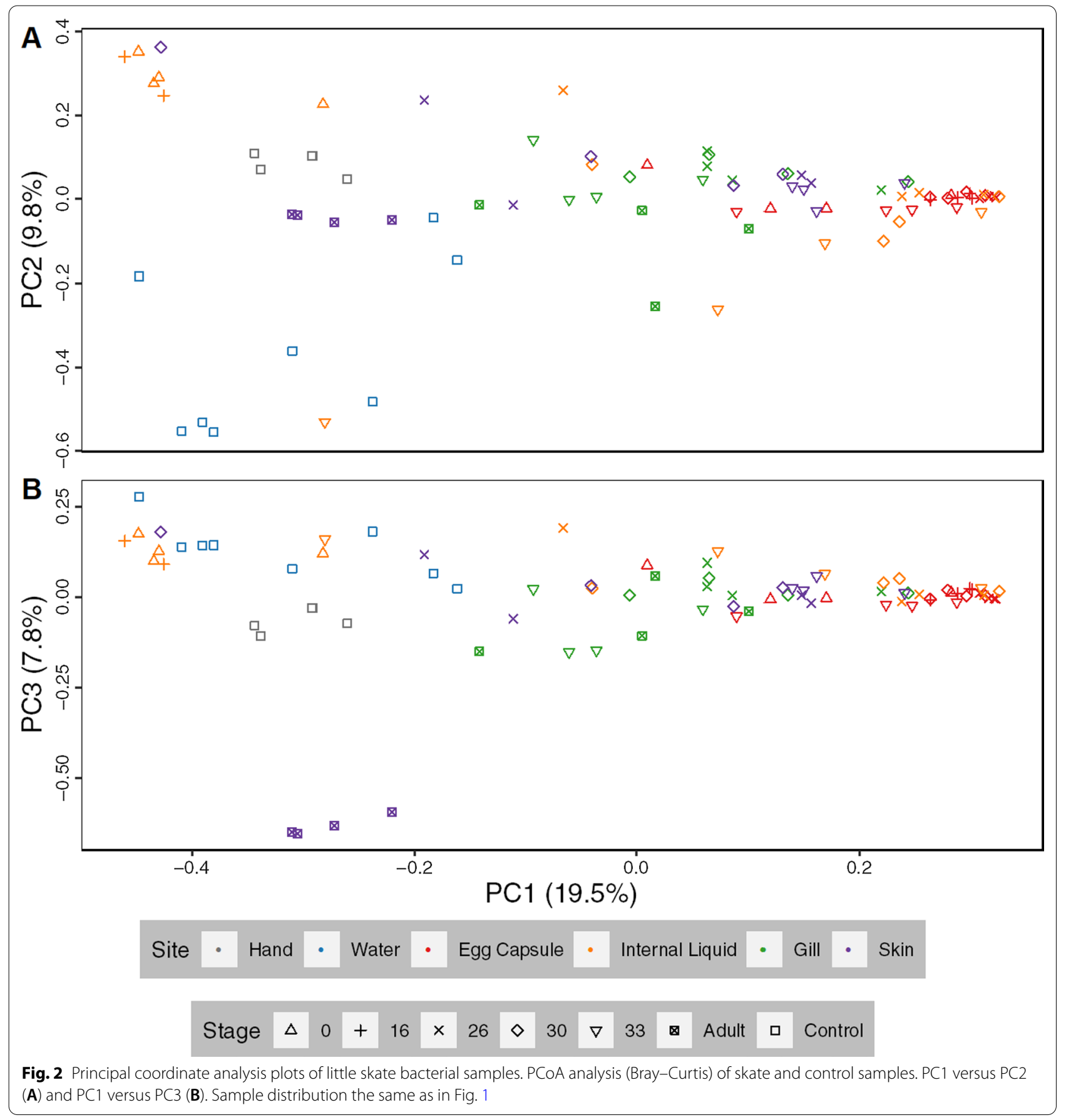


community, while in all other samples Proteobacteria are most abundant. Within the control samples, the water samples are dominated by Proteobacteria (87\%), followed by Bacteroidetes (5\%), Cyanobacteria (2.6\%), and Actinobacteria (2\%). The investigators' hand samples are quite distinct from all other samples, dominated by the phyla Actinobacteria (49\%), Cyanobacteria (31\%), Proteobacteria (9\%) and Firmicutes (5\%), showing the characteristic microbial community skew associated with ocean water exposure [55]. As is typical when investigating poorly studied environments, fewer ASVs can be categorized at finer phylogenetic resolutions, with $<98 \%$ being classified at the phylum level, $97 \%$ at the class level, $<83 \%$ at the order level, $<70 \%$ at the family level and $<60 \%$ at the genus level. The most common families identified are Rhodobacteraceae (8.1\%), Flavobacteriaceae (6.9\%), Enterobacteraceae (6.1\%), Saprospiraceae (3.2\%), and Devosiaceae (3.2\%) (Additional file 4: Fig. 3). The most common genera are Escherichia-Shigella (6.1\%), Cutibacterium (3.2\%), Devosia (2.0\%), and Lutibacter (0.9\%) (Additional file 4: Fig. 4).

\section{Beta and alpha diversity of skate bacterial communities}

We explored variation in microbial community composition between samples-Beta diversity-by sample tissue and stage using Bray-Curtis dissimilarity (Fig. 2). Due to the low number of internal liquid samples at stage 16 which successfully amplified $(n=2)$, these samples cannot be statistically differentiated from other samples but cluster tightly with stage 0 internal liquid. Water is likewise distinct from all skate tissues $(\mathrm{q}<0.05)$ except internal liquid from stage 33 embryos $(q<0.08)$, at which time the internal liquid contains a significant amount of water due to the egg capsule opening. All egg capsule samples form a tight cluster distinct $(q<0.08)$ from all other samples except mid-stage internal liquid (stages 16-30; $\mathrm{q}<0.50)$. Gill tissue samples split into two clusters $(\mathrm{q}<0.05)$ : the external, embryonic gill stages $(26-$ 30 ), and the internal, later stages (33-Adult). Internal gill samples are distinct from egg capsule $(\mathrm{q}<0.05)$ and late skin samples (stage 33-Adult, $\mathrm{q}<0.05$ ) while external gill samples (stage 26-30) cannot be distinguished from early skin samples (stages 26-30; q >0.20). Stage 30, 33 and adult skin are all significantly different $(\mathrm{q}<0.05)$ from each other. Adult skin is distinct from all other samples $(\mathrm{q}<0.05)$ and separates from all others along PC3 (Fig. 2B). Samples at stage 30 show no clear separation on open or closed status for any of the tissues examined.

Principal coordinate axes generated using the full dataset are primarily driven by the differences between sampling locations, which may obscure tissue-specific clustering patterns. Therefore, we stratified the data into individual tissues and reran Bray-Curtis dissimilarity analyses on the reduced datasets (Additional file 4: Fig. 5). Egg capsule samples, which have indistinct subclustering in Fig. 2, show more substructure when analyzed independently, with stages 0,30 , and 33 forming unique subclusters $(\mathrm{q}<0.05$; Additional file 4: Fig. 5A). Internal liquid has no statistically significant subclusters $(q>0.09$; Additional file 4: Fig. 5B). Bray-Curtis dissimilarity analysis on all gill samples supports the internal and external gill subclusters seen in the full dataset (Additional file 4: Fig. $5 \mathrm{C} ; \mathrm{q}<0.05)$. Adult skin is distinct from all other skin (Additional file 4: Fig. 5D; $\mathrm{q}<0.05$ ) along PC1 which explains $36.5 \%$ of the variance within skin samples. Due to high variability in stage 26 and 30 skin, stage 33 skin is statistically differentiated $(\mathrm{q}<0.05)$ but clusters tightly with the majority of these samples.

We explored diversity within a sample (alpha diversity), in two different ways: we used the Shannon index to assess both observed richness (the observed number of ASVs present in each sample) and evenness (relative abundance of ASVs in each sample): and the Chao1 index to assess the estimated total richness of the tissue site (the number of ASVs in the population represented by each sample) (Fig. 3). Total ASV counts and Pielou's evenness for each sample are listed in Additional file 2: Table S2. Shannon and Chaol indices were calculated for each sample site, splitting gills into external (stages 26-30) and internal (stages 33-Adult), skin into embryonic (stages 26-33) and adult, and averaging across all stages for egg capsule and internal liquid. The total richness of the egg capsule is significantly greater than all other sampling sites by Chao1 (pairwise Kruskal-Wallis, $\mathrm{q}<0.05$ ). The total richness of the internal liquid increases with stage, but this trend is not significant; a similar trend is not seen in the egg capsule. When considering observed richness and evenness, egg capsule samples have a significantly higher mean Shannon index than all other sites except external gills. Taken together this suggests that while external gills have a relatively small number of taxa, the community is much more evenly distributed than most other sites. In contrast, adult skin has a small number of taxa dominated by a few highly abundant taxa. No other pairwise comparisons were significant using either metric.

\section{Microbial source identification for each stage}

We performed FEAST source tracking on each stage to assess the relative contribution of each sampling site in facilitating intergenerational transmission. FEAST estimates the relative proportional contribution of each potential source and assigns any unexplained components of the sink bacterial community to an unknown source [52]. A consequence of this emphasis on proportional mixing is limited power to identify the source of 

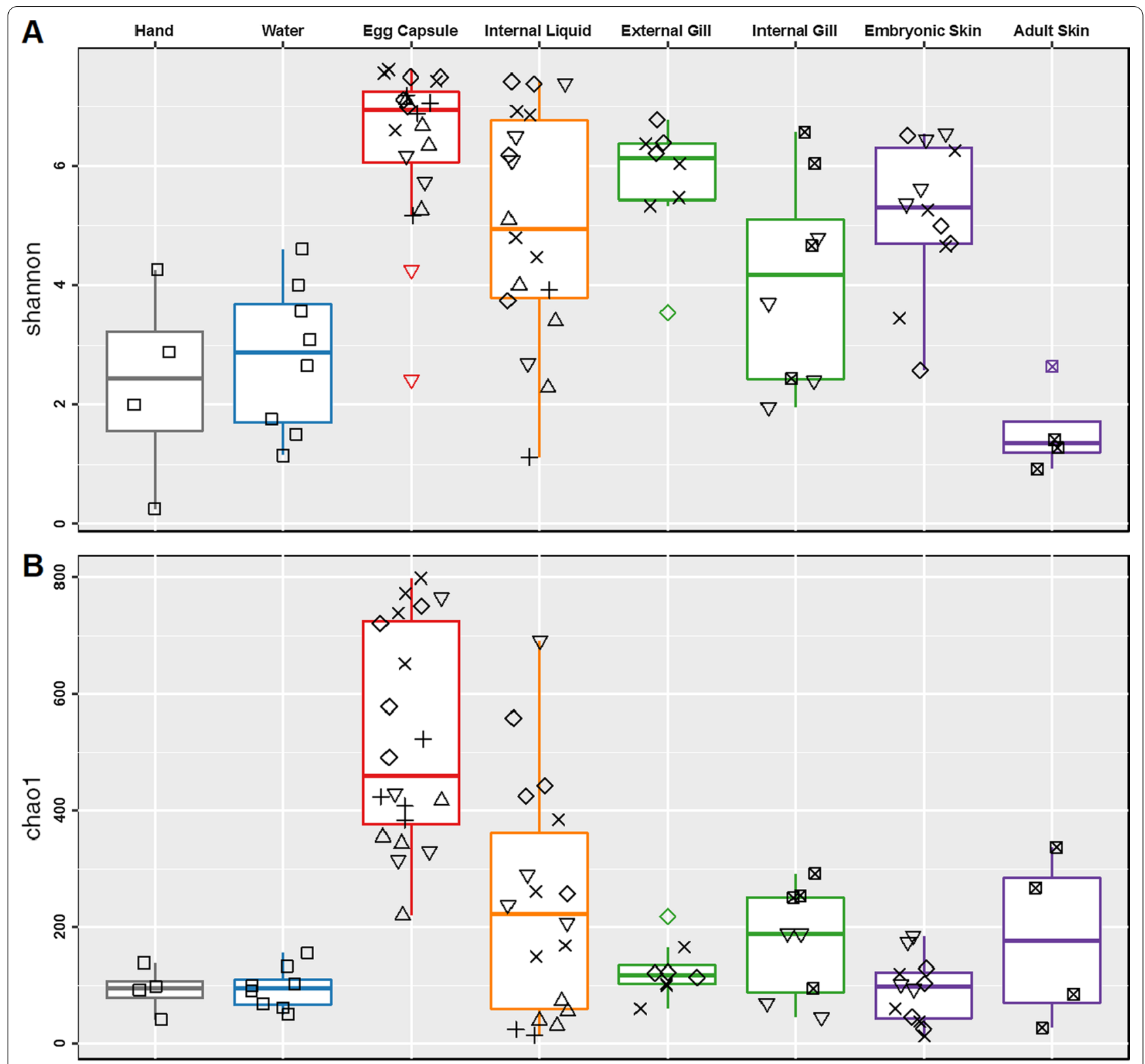

Stage $\Delta 0+16 \times 26 \diamond 30 \nabla 33 \quad$ Adult $\square$ Control

Fig. 3 Alpha diversity of embryonic and adult skate bacterial communities. Boxplots of Shannon (A) and Chao1 (B) alpha diversity metrics for each sample site. Datapoints shown in black. Colored points are statistical outliers. Stage is indicated by shape. Grey: experimenters' hands, blue: water, red: egg capsule, orange: internal liquid, green: gill, and purple: skin

taxa with higher relative abundances in the sink compared to any source, leading to inflated unknown source values. Since some taxa are likely enriched during development of the little skate, FEAST values should be interpreted as identifying the most important sources at each stage, not directly estimating continuity between timepoints. Samples from the preceding embryonic stage were used as source pools for the target community (sink) of the focal stage to track microbial continuity until hatching. At stage 0 , swabs from the representative adult females were used as the source pools (Fig. 4). For all timepoints, water samples were included as an environmental source pool, and the experimenters' hands were also considered as a source of potential contamination.

Hand samples are a negligible source for all samples except stage 0 internal liquid (hand contribution 


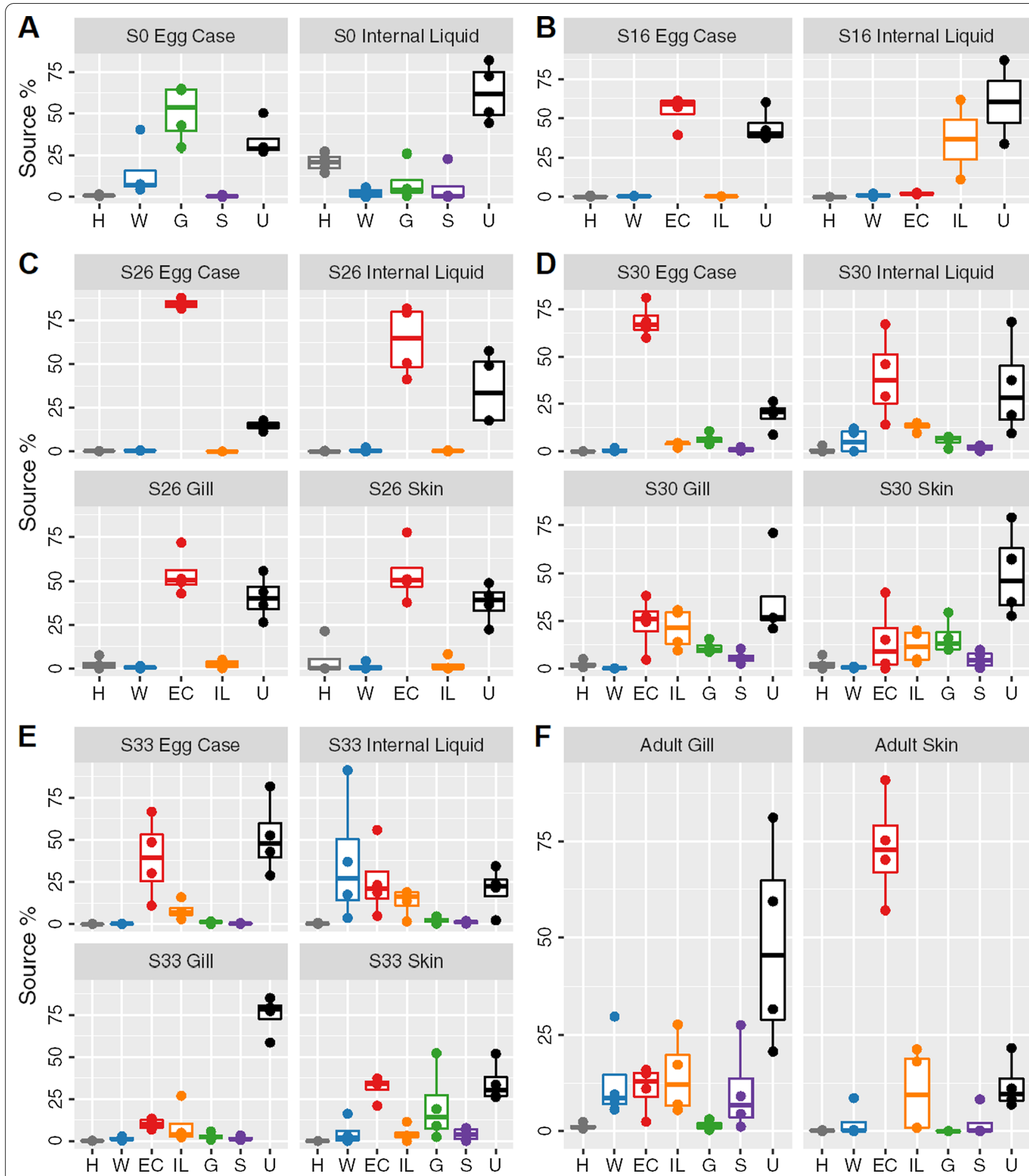

Fig. 4 Source contributions to the little skate microbiota for each stage and tissue. Boxplots showing source contributions to the bacterial community of the skate microbiota at stage $0(\mathbf{A})$, stage $16(\mathbf{B})$, stage $26(\mathbf{C})$, stage 30 (D), stage $33(\mathbf{E})$ and adult (F) estimated using FEAST. Stage 0 used adult tissues as the source pools. Source contributions to adults $(\mathbf{F})$ are shown for stage 33 source pools. Sources are colored as in Fig. 2. Letter codes refer to source pools from the previous stage or controls. H: Experimenters' hands, W: water; EC: egg capsule, IL: egg capsule internal liquid, G: gill, S: skin, U: unknown source 
$20.1 \pm 5.5 \%$, mean \pm standard deviation, Fig. 4 A). Environmental water rarely contributes to the little skate microbiota; the exceptions are stage 0 egg capsule $(14.8 \pm 16.9 \%)$ and stage 33 internal liquid (37.3 $\pm 38.5 \%)$. In addition to water, the egg capsule microbiota at stage 0 is similar to the adult gill community $(50.2 \pm 17.2 \%$, Fig. 4A). At stage 16, the egg capsule and internal liquid microbiota are both largely conserved from the same tissue of the previous stage $(54.6 \pm 10.4 \%$ and $36.5 \pm 35.7 \%$ continuity, respectively, Fig. 4B). At stage 26, egg capsule from the previous stage is the dominant bacterial source for all tissues $(63.9 \pm 18.4 \%$, Fig. 4 C). At stage 30 , egg capsule and internal liquid are sourced primarily from stage 26 egg capsule $(68.6 \pm 8.9 \%$ and $39.0 \pm 22.7 \%$, respectively), but gill and skin are sourced from a combination of the egg capsule, internal liquid, and gill of the previous stage (Fig. 4D). At stage 33, egg capsule remains contiguous $(39.1 \pm 24.0 \%)$ while the internal liquid draws from egg capsule $(25.6 \pm 21.6 \%)$ and internal liquid $(13.2 \pm 8.2 \%)$ sources along with environmental water (Fig. 4E). The stage 33 gill microbiota, comprised of the earliest internal gill samples, is not particularly similar to any source while the skin is most similar to S30 egg capsule $(31.6 \pm 7.2 \%)$.

To understand the extent to which taxa from the embryonic microbiota contributes to the adult bacterial communities, we ran FEAST on the adult samples using stage 33 tissues as the source pools (Fig. 4F, top). Adult gill has some contribution from stage 33 egg capsule $(11.1 \pm 6.1 \%)$, internal liquid $(14.3 \pm 10.3 \%)$, and skin $(10.6 \pm 11.8 \%)$. The adult skin bacterial community is primarily derived from that of the late embryonic egg capsule $(73.3 \pm 14.0 \%)$ (Fig. 4F).

\section{Identification of skate core microbiota}

For subsequent analyses, we used Bray-Curtis dissimilarity to group samples of interest into four statistically significant and biological relevant groups: (1) egg capsule (all stages), (2) external gills (stages 26-30) and embryonic skin (stages 26-33) together, (3) internal gill (stage 33-adult), and (4) adult skin. Due to heterogeneity in the composition of the internal liquid throughout ontogeny and lack of large source contributions to other tissues, these samples are not considered in further analyses.

To extract the core microbiota of the little skate from our dataset, we identified taxonomic groups classified to the most specific level possible which were present in $75 \%$ or more of the samples comprising the four groups specified above (Additional file 3: Table S3). Egg capsule samples have a rich core microbial community with 212 identified taxonomic groups. Skate samples have smaller core microbiota: combined external gill and embryonic skin have ten, internal gills have twenty-two, and adult skin has fifty-six groups. There is high overlap between the core microbiota of these tissues (Table 1) and only egg capsule and adult skin house unique taxa (Additional file 3: Table S3).

We cross-referenced the genera identified as core to only the adult skin with the full taxa list for all samples in order to identify taxa exclusively present on adult tissues. This comparison identified six exclusively adult taxa. Undibacterium is the only genus found in all adult skin and gill samples that does not appear in any egg capsule or embryonic sample. Spiroplasma, Salimicrobium, and Proprionivibrio were each found in a single adult gill sample and in the adult skin core microbiota. Sulfurospirillum sp. SM-5 and Aeromonas were unique to adult skin tissues.

\section{Differential abundance of bacterial taxa}

For each of the four sample groups in Table 1, we used LEfSe analysis to identify taxa enriched in abundance $(P<0.05$, LDA $>2$; Additional file 4: Fig. 6). Adult skin is enriched in Bacteroidia, Vibrio, and Mycoplasma agassizii. Embryonic skin and the external gills are enriched in Sphingomondales, Flavobacteriaceae, and EscheriaShigella. Internal gills are enriched in Rhodobacterales and Alphaproteobacteria of SAR11 clade 1. Finally, the egg capsule is characterized by higher abundance of Alteromonadales, Pirellulales, Saprospiraceae, and Verrucomicrobia.

Additionally, we used LEfSe to identify shifts in taxa abundance associated with the opening of the egg capsule slits. We compared open and closed samples of the egg capsule, internal liquid, and a combined set of the two tissues. While a few taxa were identified as statistically significant, closer inspection of abundances in each sample did not exhibit the expected pattern of similar levels of abundance across all samples in one condition compared to the other. Instead, significance was driven by differences in group means due to a few samples with high abundances in either the open or closed condition (Additional file 4: Fig. 7).

\section{Discussion}

Our results show that the phyla Proteobacteria and Bacteroidetes comprise most of the bacterial communities associated with the little skate, as has been shown for other chondrichthyans $[8,9,11-13]$. Adult skate skin within our study has a uniquely high proportion of $\mathrm{Bac}$ teroidetes (>50\%) compared to all other batoids [11, 12]. Within Proteobacteria, relative proportions of each class vary between chondrichthyan species, including the little skate $[8,9,11-13]$. Below the phylum-level, there is evidence of unique site-specific communities in our study. While all skate samples included Gammaproteobacteria, 
Table 1 Core taxonomic units shared between tissues

\begin{tabular}{|c|c|c|c|c|}
\hline Taxa & Egg capsule & $\begin{array}{l}\text { External gill/embryonic } \\
\text { skin }\end{array}$ & Internal gill & Adult skin \\
\hline Actinomarinales & $x$ & & & $x$ \\
\hline Alphaproteobacteria & $x$ & $x$ & $x$ & $x$ \\
\hline Bacteroidia & $x$ & $x$ & & $x$ \\
\hline Burkholderiaceae & $x$ & & & $x$ \\
\hline Candidatus Nitrosopumilus & $x$ & & $x$ & \\
\hline Chloroplast & $x$ & & $x$ & $x$ \\
\hline Colwellia & $x$ & & $x$ & $x$ \\
\hline Escherichia-Shigella & $x$ & $x$ & $x$ & \\
\hline Flavobacteriaceae & $x$ & $x$ & $x$ & $x$ \\
\hline Flavobacteriales & $x$ & & & $x$ \\
\hline Gammaproteobacteria & $x$ & $x$ & $x$ & $x$ \\
\hline Halieaceae & $x$ & $x$ & & $x$ \\
\hline Lentisphaera & $x$ & & $x$ & $x$ \\
\hline Pirellulaceae & $x$ & $x$ & $x$ & $x$ \\
\hline Pseudoalteromonas & $x$ & & $x$ & $x$ \\
\hline Pseudomonas & $x$ & & & $x$ \\
\hline Rhizobiaceae & $x$ & & $x$ & $x$ \\
\hline Rhodobacteraceae & $x$ & $x$ & $x$ & $x$ \\
\hline Rhodothermaceae & $x$ & & & $x$ \\
\hline Rubritalea & $x$ & & & $x$ \\
\hline Saprospiraceae & $x$ & $x$ & $x$ & $x$ \\
\hline SAR11 clade (Clade I) & $x$ & & $x$ & $x$ \\
\hline Shewanella & $x$ & & $x$ & \\
\hline Sphingomonadaceae & $x$ & & $x$ & $x$ \\
\hline Sulfitobacter & $x$ & & & $x$ \\
\hline Synechococcus CC9902 & $x$ & & $x$ & \\
\hline Thermoanaerobaculaceae Subgroup 10 & $x$ & & & $x$ \\
\hline Thiothrix sp. FBR0112 & $x$ & & & $x$ \\
\hline Ulvibacter & $x$ & & $x$ & \\
\hline Uncultured verrucomicrobium DEVOO7 & $x$ & & $x$ & \\
\hline Verrucomicrobium sp. KLE1210 & $x$ & & & $x$ \\
\hline Vibrio & $x$ & & & $x$ \\
\hline Vibrionaceae & $x$ & & $x$ & $x$ \\
\hline
\end{tabular}

List of taxa identified as part of the common core microbiota at a $75 \%$ presence cut-off in a least two of the following tissues: egg capsule, combined external gill (stages 26-30) and embryonic skin, internal gill (stage 33-adult), and adult skin

Flavobacteriaceae, Pirellulaceae, Rhodobacteraceae, and Saprospiraceae, we are unable to identify a common core microbial community below the family level for all skate samples across all developmental timepoints. Instead, our data suggest that early embryonic tissues support similar bacterial communities which differentiate into distinct internal gill and adult skin microbial communities later in development.

We sampled two parts of the egg capsule: the inner surface of the capsule and the internal liquid, which fills the space in the egg capsule not occupied by the developing embryo and yolk. The microbiota of the egg capsule has the highest taxonomic richness of any tissue sampled and has a complex core microbiota. This provides evidence that the egg capsule is a rich reservoir of bacteria for the developing embryo, similar to the dense microbial community observed in squid egg capsules $[56,57]$. While the microbiota of the internal liquid is generally similar to egg capsule samples, there appears to be a collapse of Actinobacteria and Bacilli after stage 16 , both rare in the egg capsule, with relative replacement by Bacteriodia and Planctomycetacia, both more abundant in the egg capsule. No taxa 
undergo consistent shifts in abundance in either the egg capsule or internal liquid upon opening.

Gills are the primary site of gas and waste exchange with the environment, offering a unique habitat for microbes. Little skate embryos develop transient external gill filaments between stages 25 and 32, which later regress into the body to form the adult internal gills [36]. During early stages, we find that the gill microbial community is undifferentiated from that of the embryonic skin. The mature gills, however, harbor a distinct microbial community, which is enriched for Rhodobacterales and Alphaproteobacteria of the SAR11 clade. These taxa are also enriched in the gills of reef fish, suggesting that marine vertebrate gills provide similar microbial environments [58]. Additionally, gills are the largest site of urea loss in skates [59]. This makes adult gill tissue particularly well-suited for a commensal relationship with Nitrosopumilus, which is known to use urea as an energy source [60], and is identified as a core bacterial genus in the gills starting at stage 33 .

Adult skin has the lowest Shannon diversity of any tissue and unlike all other sites its microbiota is primarily composed of Bacteroidetes ASVs assigned to class Bacteroidia (81.2\%). Previous work has shown that Bacteroidetes are dominant in many niches, are adapted to life on marine surfaces, play roles in polymer degradation, and contribute to immune function [61, 62]. Given the abundance of Bacteroidetes on skate skin, the functional implications of these ASVs on the host is a promising area for future research.

The low diversity observed in the adult skin samples may be due to the distinct properties of chondrichthyan skin, which is characterized by dermal denticles and a thin mucus layer [63]. Since the denticles do not develop until around the time of hatching, the biophysical properties of skate skin change after embryonic development is complete [64]. The skin is hypothesized to offer a selective microbial environment due to micropatterning of the dermal denticles, reduced laminar flow, and antimicrobial compounds $[9,65]$. In support of this hypothesis, shark skin micropatterning has been shown to hinder microbial colonization and migration [66, 67]. Our data show that the adult skate skin bacterial community is distinct from that of embryonic skin, which clusters closely with the other embryonic tissues (Fig. 2). We hypothesize that the development of mature denticles shapes the bacterial community of adult chondrichthyan skin, though this hypothesis requires explicit testing. An alternate possibility for the low-diversity skin microbiota is that the taxa present possess antimicrobial properties and suppress competition. While stingray skin microbiota show some antibiotic potential, skate skin microbes exhibit lower levels of antibiotic activity $[68,69]$. Future work is needed to assess the relative contributions of biophysical properties, antimicrobial secretions, and other mechanisms in shaping the bacterial community on chondrichthyan skin and the relevance of this community to host physiology and health.

Environmental sources likely contribute to the microbiota of adult skates. The one environmental source included in this study, the surrounding water, was not found to contribute meaningfully to gills or skin at any stage, however, deeper sampling may be necessary to detect low abundance taxa present in the water column. Identifying other potential environmental sources, such as diet and benthic substrates, for wild caught adults is not feasible and these sources could not be sampled for this study. Taxa identified only on adults cannot be explained by vertical transmission. In this study, we identified only six genera uniquely present on adult tissues. Of these six, only a single genus, Undibacterium, was present in all adult samples of both skin and gill. Undibacterium species have been isolated from other fishes and may play a role in biofilm degradation [70-73]. Given that this taxon was not detected in embryonic or water samples, skates likely acquire Undibacterium from an unknown environmental source, drastically enrich this genus from starting levels below the limit of detection, or through horizontal transmission.

We provide evidence of vertical transmission in an elasmobranch by tracking community continuity between different developmental stages and tissues. First, we found minimal water contributions to each sample, with the exception of internal liquid at stage 0 and after egg capsule opening. Second, skate samples have largely similar taxa between consecutive stages, with the largest shifts in community composition matching developmental changes in the physical properties of host tissues. Given the sealed nature of the egg capsule, differences in community composition are hypothesized to reflect enrichment of existing bacteria rather than recruitment from unknown sources, but this requires additional study to confirm. Finally, only six taxa were exclusively found on adult tissues, suggesting environmental sourcing may be limited. While these points are insufficient to show vertical transmission, taken together, along with the life history of the little skate, they imply vertical microbial transmission occurs. If future studies identify vertical transmission in this and additional chondrichthyan species, particularly those with alternate life histories, this would provide a potential mechanism underlying the signature of phylosymbiosis observed by others in elasmobranchs [11].

There are additional considerations for this dataset given our experimental design. Since individual egg capsules were not associated with particular adult females, 
direct pairwise comparisons between parent and offspring were not possible. Furthermore, embryonic sampling is lethal, so each developmental timepoint is comprised of different, unrelated embryos. Thus, interindividual variation limits our ability to accurately track all ASVs between timepoints. While we did sample adult female skin at the cloaca, these samples are unlikely to capture the extent of diversity housed in the reproductive tract where the egg capsules form [25]. Like most elasmobranchs, little skates are polyandrous and multiple paternity is likely [74]. Similar promiscuity has been associated with higher microbial diversity in the female reproductive tract in other vertebrate groups [75-77], a pattern that may hold for skates. We hypothesize that the microbiota of the reproductive tract is highly diverse, potentially providing a richer source of microbiota to the egg capsule than is captured by the adult tissues sampled in this study. Nonetheless, the precise mechanisms by which the egg capsule is seeded with its rich bacterial community, and how transmission of pathogenic bacteria is minimized, await further investigation.

\section{Conclusions}

This study provides the first exploration of the bacterial communities associated with the little skate throughout ontogeny and offers many intriguing possibilities for future microbiome research using this model chondrichthyan. Specifically, we identified a site-specific microbiota that is likely transferred between generations via the internal surface of the egg capsule and provide the first evidence that vertical transmission is present in an oviparous elasmobranch species.

\section{Abbreviations}

MRC: Marine Resources Center at the Marine Biological Laboratory, Woods Hole, MA, USA.; ASV: Amplicon sequencing variant; FDR: False discovery rate; LDA: Linear discriminant analysis; PC: Principal coordinate.

\section{Supplementary Information}

The online version contains supplementary material available at https://doi. org/10.1186/s42523-021-00136-x.

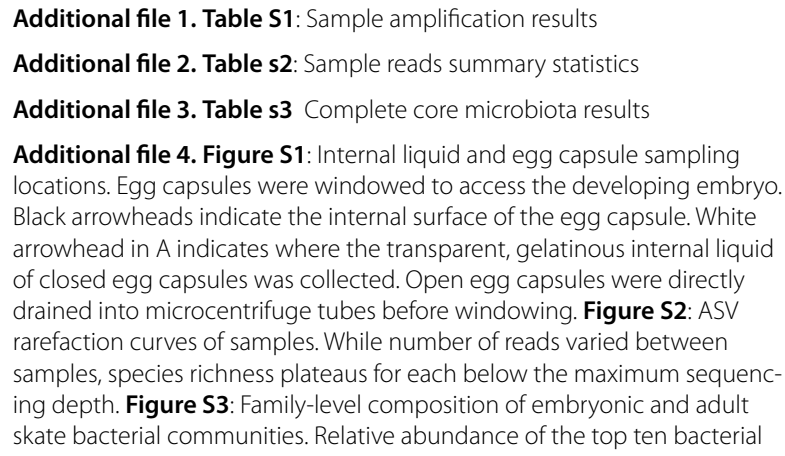

families in the classes Alphaproteobacteria (A), Gammaproteobacteria (B), and Bacteroidia (C) in the dataset are shown for each site and timepoint as well as for water and hand controls. Figure S4: Genus-level composition of embryonic and adult skate bacterial communities. Relative abundance of the top ten bacterial genera in the classes Alphaproteobacteria (A), Gammaproteobacteria (B), and Bacteroidia (C) in the dataset are shown for each site and timepoint as well as for water and hand controls. Figure S5: Principal coordinate analysis plots of bacterial communities by tissue. PCoA analysis (Bray-Curtis) plots of PC1 versus PC2 for (A) egg capsule, (B) Internal liquid, (C) gill, and (D) skin samples. Figure S6: Differentially abundant bacterial taxa among skate tissues. LEfSe analysis at $\mathrm{P}<0.05$ and LDA>2. Only differentially abundant tree branches are shown. eggcase: egg capsule, Egillskin: embryonic external gills (stages 16-30) and embryonic skin (stages 16-33), Lgill: internal gill (stage 33-Adult), adultskin: adult skin. Figure S7: Significant taxa comparisons in closed versus open egg capsules. Heatmap of the taxa identified by LEfSe $(P<0.05, L D A>2)$ at the lowest categorized taxonomic level for comparisons of open versus closed egg capsule (red), internal liquid (orange) and combined egg capsule and internal liquid (black). Abundances of each sample are shown as Z-scores. Open samples include stage 30 replicates A \& D, as well as all stage 33 samples. All other samples come from closed egg capsules.

\section{Acknowledgements}

The authors thank David Remsen, Dan Calzarette, and the MRC staff for assistance sampling the skates; Andrew Gillis for inspiration and staging the skate embryos, Tetsuya Nakamura for assistance with our IACUC protocol, Emily Davenport for directing us to computational resources, and Nipam H. Patel for use of lab space. We are deeply grateful to Tom A. Stewart, Terence D. Capellini, and the Carmody Lab for helpful comments on the manuscript.

\section{Authors' contributions}

K.M. and A.S.O. conceived of and designed the project in consultation with N.H.S and D.M.W. K.M. and A.S.O. collected the data, performed the computational analyses, and wrote the manuscript. All authors edited and approved the final paper.

\section{Funding}

This project was funded by a Microbiome Pilot Project Grant from the Microbiome Center of the University of Chicago, Marine Biological Laboratory, and Argonne National Laboratory to D.M.W and N.H.S. This material is based upon work supported by the National Science Foundation Graduate Research Fellowship to A.S.O. (DGE1745303).

\section{Availability of data and materials}

Raw sequencing data is available at the NCBI Short Read Archive (PRJNA688288). All scripts are available online at https://github.com/kmmika/ Intergenerational-microbial-transmission-in-the-little-skate-Leucoraja-erina cea.

\section{Declarations}

\section{Ethics approval}

All procedures were conducted in accordance with Marine Biological Laboratory IACUC protocol 19-42.

\section{Consent for publication}

Not applicable.

\section{Competing interests}

The authors declare no competing interests.

\section{Author details}

${ }^{1}$ Organismal Biology and Anatomy, University of Chicago, 900 E 57th St, Culver Hall 108 OBA, Chicago, IL 60637-1428, USA. ${ }^{2}$ Genetic Medicine, University of Chicago, Chicago, USA. ${ }^{3}$ Human Evolutionary Biology, Harvard University, Cambridge, MA, USA. ${ }^{4}$ Josephine Bay Paul Center for Comparative Molecular Biology and Evolution, Marine Biological Laboratory, Woods Hole, MA, USA. 
Received: 25 June 2021 Accepted: 26 September 2021

Published online: 13 October 2021

\section{References}

1. Chiarello M, Villéger S, Bouvier C, Auguet JC, Bouvier T. Captive bottlenose dolphins and killer whales harbor a species-specific skin microbiota that varies among individuals. Sci Rep. 2017;7:15269. https://doi.org/10.1038/ s41598-017-15220-Z.

2. Larsen A, Tao Z, Bullard SA, Arias CR. Diversity of the skin microbiota of fishes: evidence for host species specificity. FEMS Microbiol Ecol. 2013:85:483-94. https://doi.org/10.1111/1574-6941.12136.

3. Leftwich PT, Edgington MP, Chapman T. Transmission efficiency drives host-microbe associations. Proc R Soc B Biol Sci. 2020;287:20200820. https://doi.org/10.1098/rspb.2020.0820.

4. Funkhouser LJ, Bordenstein SR. Mom knows best: the universality of maternal microbial transmission. PLoS Biol. 2013;11:e1001631. https://doi. org/10.1371/journal.pbio.1001631

5. Metcalf CJE, Henry LP, Rebolleda-Gómez M, Koskella B. Why evolve reliance on the microbiome for timing of ontogeny? MBio. 2019;10:e0149619. https://doi.org/10.1128/mBio.01496-19.

6. Compagno LJV. Alternative life-history styles of cartilaginous fishes in time and space. Environ Biol Fishes. 1990;28:33-75. https://doi.org/10. 1007/BF00751027.

7. Givens CE, Ransom B, Bano N, Hollibaugh JT. Comparison of the gut microbiomes of 12 bony fish and 3 shark species. Mar Ecol Prog Ser. 2015;518:209-23. https://doi.org/10.3354/meps11034.

8. Pogoreutz C, Gore MA, Perna G, Millar C, Nestler R, Ormond RF, et al. Similar bacterial communities on healthy and injured skin of black tip reef sharks. Anim Microbiome. 2019;1:9. https://doi.org/10.1186/ s42523-019-0011-5.

9. Doane MP, Haggerty JM, Kacev D, Papudeshi B, Dinsdale EA. The skin microbiome of the common thresher shark (Alopias vulpinus) has low taxonomic and gene function $\beta$-diversity. Environ Microbiol Rep. 2017;9:357-73. https://doi.org/10.1111/1758-2229.12537.

10. Johny TK, Saidumohamed BE, Sasidharan RS, Bhat SG. Metabarcoding data of bacterial diversity of the deep sea shark, Centroscyllium fabricii. Data Br. 2018;21:1029-32. https://doi.org/10.1016/j.dib.2018.10.062.

11. Doane MP, Morris MM, Papudeshi B, Allen L, Pande D, Haggerty JM, et al The skin microbiome of elasmobranchs follows phylosymbiosis, but in teleost fishes, the microbiomes converge. Microbiome. 2020;8:93. https:// doi.org/10.1186/s40168-020-00840-x.

12. Kearns PJ, Bowen JL, Tlusty MF. The skin microbiome of cow-nose rays (Rhinoptera bonasus) in an aquarium touch-tank exhibit. Zoo Biol. 2017:36:226-30. https://doi.org/10.1002/zoo.21362.

13. Storo R, Easson C, Shivji M, Lopez JV. Microbiome Analyses Demonstrate Specific Communities Within Five Shark Species. Front Microbiol. 2021;12:605285. https://doi.org/10.3389/fmicb.2021.605285.

14. Apprill A, Robbins J, Eren AM, Pack AA, Reveillaud J, Mattila D, et al. Humpback whale populations share a core skin bacterial community: Towards a health index for marine mammals? PLoS One. 2014;9:e90785. https://doi.org/10.1371/journal.pone.0090785.

15. Wahl M, Goecke F, Labes A, Dobretsov S, Weinberger F. The second skin: Ecological role of epibiotic biofilms on marine organisms. Front Microbiol. 2012;3:292. https://doi.org/10.3389/fmicb.2012.00292.

16. Chiarello $M$, Villéger $S$, Bouvier $C$, Bettarel $Y$, Bouvier T. High diversity of skin-associated bacterial communities of marine fishes is promoted by their high variability among body parts, individuals and species. FEMS Microbiol Ecol. 2015;91:fiv061. https://doi.org/10.1093/femsec/fiv061.

17. Lim SJ, Bordenstein SR. An introduction to phylosymbiosis. Proc R Soc B Biol Sci. 2020:287:20192900. https://doi.org/10.1098/rspb.2019.2900.

18. Jacoby DMP, Croft DP, Sims DW. Social behaviour in sharks and rays: analysis, patterns and implications for conservation. Fish Fish. 2012;13:399-417. https://doi.org/10.1111/j.1467-2979.2011.00436.x.

19. Pratt HL, Carrier JC. A review of elasmobranch reproductive behavior with a case study on the nurse shark, Ginglymostoma cirratum. Environ Biol Fishes. 2001;60:157-88. https://doi.org/10.1023/A:1007656126281.

20. Dulvy NK, Reynolds JD. Evolutionary transitions among egg-laying, live-bearing and maternal inputs in sharks and rays. Proc R Soc B Biol Sci. 1997;264:1309-15. https://doi.org/10.1098/rspb.1997.0181.
21. Chiquillo KL, Ebert DA, Slager CJ, Crow KD. The secret of the mermaid's purse: phylogenetic affinities within the Rajidae and the evolution of a novel reproductive strategy in skates. Mol Phylogenet Evol. 2014;75:24551. https://doi.org/10.1016/j.ympev.2014.01.012.

22. Hobson AD. A note on the formation of the egg case of the skate. J Mar Biol Assoc United Kingdom. 1930;16:577-81. https://doi.org/10.1017/ S0025315400072945.

23. Palm BD, Koester DM, Driggers WB, Sulikowski JA. Seasonal variation in fecundity, egg case viability, gestation, and neonate size for little skates, Leucoraja erinacea, in the Gulf of Maine. Environ Biol Fishes. 2011;92:585-89.

24. Di Santo V. Ocean acidification exacerbates the impacts of global warming on embryonic little skate, Leucoraja erinacea (Mitchill). J Exp Mar Bio Ecol. 2015;463:72-8. https://doi.org/10.1016/j.jembe.2014.11.006.

25. Serra-Pereira B, Figueiredo I, Gordo LS. Maturation of the gonads and reproductive tracts of the thornback Ray Raja Clavata, with comments on the development of a standardized reproductive terminology for oviparous elasmobranchs. Mar Coast Fish. 2011;3:160-75. https://doi.org/ 10.1080/19425120.2011.555707.

26. Lombardi J, Files T. Egg capsule structure and permeability in the viviparous shark. Mustelus canis J Exp Zool. 1993;267:76-85. https://doi.org/10. 1002/jez.1402670111.

27. Hornsey DJ. Permeability coefficients of the egg-case membrane of Scyliorhinus canicula L. Experientia. 1978;34:1596-7. https://doi.org/10. 1007/BF02034696.

28. Koob TJ, Summers A. On the hydrodynamic share of little skate (Raja erinacea) egg capsules. Bull Mt Desert Isl Biol Lab. 1996;35:108-11.

29. Marconi A, Hancock-Ronemus A, Gillis JA. Adult chondrogenesis and spontaneous cartilage repair in the skate. Leucoraja erinacea Elife. 2020;9:e53414. https://doi.org/10.7554/eLife.53414.

30. Nakamura T, Klomp J, Pieretti J, Schneider I, Gehrke AR, Shubin NH. Molecular mechanisms underlying the exceptional adaptations of batoid fins. Proc Natl Acad Sci. 2015;112:15940-5. https://doi.org/10.1073/pnas. 1521818112.

31. Turner N, Mikalauskaite D, Barone K, Flaherty K, Senevirathne G, Adachi $\mathrm{N}$, et al. The evolutionary origins and diversity of the neuromuscular system of paired appendages in batoids. Proc R Soc B Biol Sci. 2019:286:20191571. https://doi.org/10.1098/rspb.2019.1571.

32. Criswell KE, Coates MI, Gillis JA. Embryonic development of the axial column in the little skate. Leucoraja erinacea J Morphol. 2017;278:300-20. https://doi.org/10.1002/jmor.20637.

33. Wyffels J, King BL, Vincent J, Chen C, Wu CH, Polson SW. SkateBase, an elasmobranch genome project and collection of molecular resources for chondrichthyan fishes. F1000Research. 2014;3:191. https://doi.org/10. 12688/f1000research.4996.1.

34. Packer DB, Zetlin CA, Vitaliano JJ. Essential fish habitat source document. Little Skate, Leucoraja erinacea, life history and habitat characteristics. 2003.

35. Ballard WW, Mellinger J, Lechenault $\mathrm{H}$. A series of normal stages for development of scyliorhinus canicula, the lesser spotted dogfish (Chondrichthyes: Scyliorhinidae). J Exp Zool. 1993;267:318-36.

36. Maxwell EE, Fröbisch NB, Heppleston AC. Variability and conservation in late chondrichthyan development: ontogeny of the winter skate (Leucoraja ocellata). Anat Rec. 2008;291:1079-87. https://doi.org/10.1002/ ar.20719.

37. Nelson MC, Morrison HG, Benjamino J, Grim SL, Graf J. Analysis, Optimization and Verification of Illumina-Generated $16 \mathrm{~S}$ rRNA Gene Amplicon Surveys. PLoS ONE. 2014;9: e94249. https://doi.org/10.1371/journal.pone. 0094249.

38. Huse SM, Mark Welch DB, Voorhis A, Shipunova A, Morrison HG, Eren AM, et al. VAMPS: a website for visualization and analysis of microbial population structures. MBC Bioinforma. 2014;15:41. https://doi.org/10.1186/ 1471-2105-15-41

39. Bolyen E, Rideout JR, Dillon MR, Bokulich NA, Abnet CC, Al-Ghalith GA et al. Reproducible, interactive, scalable and extensible microbiome data science using QIIME 2. Nat Biotechnol. 2019;37:852-7. https://doi.org/10. 1038/s41587-019-0209-9.

40. Callahan BJ, McMurdie PJ, Rosen MJ, Han AW, Johnson AJA, Holmes SP. DADA2: High-resolution sample inference from Illumina amplicon data. Nat Methods. 2016;13:581-3. https://doi.org/10.1038/nmeth.3869. 
41. Yilmaz P, Parfrey LW, Yarza P, Gerken J, Pruesse E, Quast C, et al. The SILVA and "All-species Living Tree Project (LTP)" taxonomic frameworks. Nucleic Acids Res. 2014;42:D643-8. https://doi.org/10.1093/nar/gkt1209.

42. Bokulich NA, Kaehler BD, Rideout JR, Dillon M, Bolyen E, Knight R, et al. Optimizing taxonomic classification of marker-gene amplicon sequences with QIIME 2's q2-feature-classifier plugin. Microbiome. 2018;6:90. https:// doi.org/10.1186/s40168-018-0470-z.

43. Pedregosa F, Varoquaux G, Gramfort A, Michel V, Thirion B, Grisel O, et al. Scikit-learn: machine learning in python. J Mach Learn Res. 2011;12:2825-30

44. McMurdie PJ, Holmes S. Phyloseq: An R Package for Reproducible Interactive Analysis and Graphics of Microbiome Census Data. PLoS One. 2013;8:e61217. https://doi.org/10.1371/journal.pone.0061217.

45. Callahan BJ, Sankaran K, Fukuyama JA, McMurdie PJ, Holmes SP. Bioconductor Workflow for Microbiome Data Analysis: from raw reads to community analyses. F1000Research. 2016:5:1492. https://doi.org/10.12688/ f1000research.8986.2.

46. Weiss S, Xu ZZ, Peddada S, Amir A, Bittinger K, Gonzalez A, et al. Normalization and microbial differential abundance strategies depend upon data characteristics. Microbiome. 2017;5:27. https://doi.org/10.1186/ s40168-017-0237-y.

47. Faith DP, Minchin PR, Belbin L. Compositional dissimilarity as a robust measure of ecological distance. Vegetatio. 1987;69:57-68. https://doi.org/ 10.1007/BF00038687.

48. Kruskal WH, Wallis WA. Use of ranks in one-criterion variance analysis. J Am Stat Assoc. 1952;47:583. https://doi.org/10.2307/2280779.

49. Anderson MJ. Permutational Multivariate Analysis of Variance (PERMANOVA). In: Wiley StatsRef: Statistics Reference Online. Chichester, UK: John Wiley \& Sons, Ltd; 2017. https://doi.org/10.1002/9781118445112. stat07841.

50. Risely A. Applying the core microbiome to understand host-microbe systems. J Anim Ecol. 2020;89:1549-58. https://doi.org/10.1111/1365-2656. 13229.

51. Segata N, Izard J, Waldron L, Gevers D, Miropolsky L, Garrett WS, et al. Metagenomic biomarker discovery and explanation. Genome Biol. 2011;12:R60. https://doi.org/10.1186/gb-2011-12-6-r60.

52. Shenhav L, Thompson M, Joseph TA, Briscoe L, Furman O, Bogumil D, et al FEAST: fast expectation-maximization for microbial source tracking. Nat Methods. 2019;16:627-32. https://doi.org/10.1038/s41592-019-0431-x.

53. R Core Team. R: A language and environment for statistical computing. 2013. http://www.r-project.org/.

54. Wickham H. ggplot2: Elegant Graphics for Data Analysis. 2016

55. Nielsen MC, Jiang SC. Alterations of the human skin microbiome after ocean water exposure. Mar Pollut Bull. 2019;145:595-603. https://doi.org/ 10.1016/j.marpolbul.2019.06.047.

56. Barbieri E, Paster BJ, Hughes D, Zurek L, Moser DP, Teske A, et al. Phylogenetic characterization of epibiotic bacteria in the accessory nidamental gland and egg capsules of the squid Loligo pealei (Cephalopoda: Loliginidae). Environ Microbiol. 2001;3:151-67. https://doi.org/10.1046/j. 1462-2920.2001.00172.x.

57. Kerwin AH, Nyholm SV. Symbiotic bacteria associated with a bobtail squid reproductive system are detectable in the environment, and stable in the host and developing eggs. Environ Microbiol. 2017;19:1463-75. https://doi.org/10.1111/1462-2920.13665.

58. Pratte ZA, Besson M, Hollman RD, Stewart FJ. The gills of reef fish support a distinct microbiome influenced by host-specific factors. Appl Environ Microbiol. 2018;84:e00063-18. https://doi.org/10.1128/AEM.00063-18.

59. Hazon N, Wells A, Pillans RD, Good JP, Anderson WG, Franklin CE. Urea based osmoregulation and endocrine control in elasmobranch fish with special reference to euryhalinity. Comp Biochem Physiol - B Biochem Mol Biol. 2003;136:685-700. https://doi.org/10.1016/s1096-4959(03)00280-X.

60. Qin W, Heal KR, Ramdasi R, Kobelt JN, Martens-Habbena W, Bertagnolli $A D$, et al. Nitrosopumilus maritimus gen. nov., sp. nov., Nitrosopumilus cobalaminigenes sp. nov., Nitrosopumilus oxyclinae sp. nov., and Nitrosopumilus ureiphilus sp. nov., four marine ammoniaoxidizing archaea of the phylum thaumarchaeo. Int J Syst Evol Microbiol. 2017;67:5067-79. https://doi.org/10.1099/ijsem.0.002416.

61. Fernández-Gómez B, Richter M, Schüler M, Pinhassi J, Acinas SG, González $J M$, et al. Ecology of marine bacteroidetes: a comparative genomics approach. ISME J. 2013;7:1026-37. https://doi.org/10.1038/ismej.2012. 169.

62. Troy EB, Kasper DL. Beneficial effects of Bacteroides fragilis polysaccharides on the immune system. Front Biosci. 2010;15:25-34. https://doi.org/ $10.2741 / 3603$.

63. Meyer W, Seegers U. Basics of skin structure and function in elasmobranchs: a review. J Fish Biol. 2012;80:1940-67. https://doi.org/10.1111/j. 1095-8649.2011.03207.x.

64. Cooper RL, Thiery AP, Fletcher AG, Delbarre DJ, Rasch LJ, Fraser GJ. An ancient Turing-like patterning mechanism regulates skin denticle development in sharks. Sci Adv. 2018;4:eaau5484. https://doi.org/10.1126/ sciadv.aau5484.

65. Lee M. Shark Skin: Taking a Bite Out of Bacteria. In: Lee M, editor. Remarkable Natural Material Surfaces and Their Engineering Potential. Cham: Springer International Publishing; 2014. https://doi.org/10.1007/ 978-3-319-03125-5 2

66. Reddy ST, Chung KK, McDaniel CJ, Darouiche RO, Landman J, Brennan AB. Micropatterned surfaces for reducing the risk of catheter-associated urinary tract infection: an in vitro study on the effect of sharklet micropatterned surfaces to inhibit bacterial colonization and migration of uropathogenic Escherichia coli. J Endourol. 2011;25:1547-52. https://doi. org/10.1089/end.2010.0611.

67. Mann EE, Manna D, Mettetal MR, May RM, Dannemiller EM, Chung KK, et al. Surface micropattern limits bacterial contamination. Antimicrob Resist Infect Control. 2014;3:28. https://doi.org/10.1186/2047-2994-3-28,

68. Ritchie KB, Schwarz M, Mueller J, Lapacek VA, Merselis D, Walsh CJ, et al. Survey of antibiotic-producing bacteria associated with the epidermal mucus layers of rays and skates. Front Microbiol. 2017;8:1050. https://doi. org/10.3389/fmicb.2017.01050.

69. Cho SH, Lee BD, An H, Eun JB. Kenojeinin I, antimicrobial peptide isolated from the skin of the fermented skate, Raja kenojei. Peptides. 2005;26:5817. https://doi.org/10.1016/j.peptides.2004.11.011.

70. Morohoshi T, Oi T, Suzuki T, Sato S. Identification and characterization of a novel extracellular polyhydroxyalkanoate depolymerase in the complete genome sequence of Undibacterium sp. KW1 and YM2 strains. PLoS One. 2020;15:e0232698. https://doi.org/10.1371/journal.pone.0232698.

71. Kämpfer P, Irgang R, Busse HJ, Poblete-Morales M, Kleinhagauer T, Glaeser SP, et al. Undibacterium danionis sp. Nov. isolated from a zebrafish (Danio rerio). Int J Syst Evol Microbiol. 2016;66:3625-31. https://doi.org/10.1099/ ijsem.0.001244.

72. Lee SY, Kang W, Kim PS, Kim HS, Sung H, Shin NR, et al. Undibacterium piscinae sp. Nov., isolated from Korean shiner intestine. Int J Syst Evol Microbiol. 2019;69:3148-54. https://doi.org/10.1099/ijsem.0.003604.

73. Lokesh J, Kiron V, Sipkema D, Fernandes JMO, Moum T. Succession of embryonic and the intestinal bacterial communities of Atlantic salmon (Salmo salar) reveals stage-specific microbial signatures. Microbiologyopen. 2019:8:e00672. https://doi.org/10.1002/mbo3.672

74. Fitzpatrick JL, Kempster RM, Daly-Engel TS, Collin SP, Evans JP. Assessing the potential for post-copulatory sexual selection in elasmobranchs. Fish Biol. 2012;80:1141-58. https://doi.org/10.1111/j.1095-8649.2012. 03256.x.

75. MacManes MD. Promiscuity in mice is associated with increased vaginal bacterial diversity. Naturwissenschaften. 2011;98:951-60. https://doi.org/ 10.1007/s00114-011-0848-2

76. White J, Richard M, Massot M, Meylan S. Cloacal bacterial diversity increases with multiple mates: Evidence of sexual transmission in female common lizards. PLoS One. 2011;6:e22339. https://doi.org/10.1371/journ al.pone.0022339.

77. Yildirim S, Yeoman CJ, Janga SC, Thomas SM, Ho M, Leigh SR, et al. Primate vaginal microbiomes exhibit species specificity without universal Lactobacillus dominance. ISME J. 2014;8:2431-44. https://doi.org/10. 1038/ismej.2014.90.

\section{Publisher's Note}

Springer Nature remains neutral with regard to jurisdictional claims in published maps and institutional affiliations. 